\title{
An Algebraic Approach to the Estimation of the Order of FIR Filters From Complete and Partial Magnitude and Phase Specifications
}

\author{
Anthony G. Constantinides, Fellow, IEEE, and Wen Min Li
}

\begin{abstract}
The problems addressed by this paper is the following: Given a set of measurements over the range of normalized frequencies $\left(\theta_{1}, \theta_{2}\right)$ on the magnitude and/or phase of a real FIR but otherwise unknown filter, to estimate the order of the FIR filter. The range $\left(\theta_{1}, \theta_{2}\right)$ may be partial or it may cover the entire range of frequencies. The purpose of the paper is to propose a new algebraic approach to solve the above collection of problems. Specific new results include FIR order estimation from partial or complete noiseless measurements for a real system from: a) phase alone, from b) magnitude alone (not necessarily piece-wise constant), and from c) joint magnitude and phase. The proposed approach is not only capable of dealing with specifications that go beyond the conventional formulas for the standard piece-wise-constant magnitude FIR filter order estimation, but it also furnishes a nexus for order estimation from phase (or group delay) specifications, areas which have remained hitherto unaddressed. The approach is based on the use of Root Moments. In this context, the novel concept of Fractional Root Moments is used in a key fashion to provide an estimate on the number of zeros inside the unit circle. Open problems and new directions of exploration and research are mentioned in the body of the paper.
\end{abstract}

Index Terms-FIR filters, fundamental relationships, Newton identities, root moments.

\section{INTRODUCTION}

$\mathbf{T}$ HE order of a FIR digital filter is always an important issue in digital filter design algorithms [1]-[5]. The paper contains new results on the following FIR order problems:

- To determine the order of a FIR filter from magnitude specifications. The standard procedure deals with piece-wise constant filter responses (the so-called band specifications) which are defined over the entire range of normalized frequencies $(0, \pi)$. There is no technique available to provide an order estimate for the nonpiece-wise constant response, or to cater for the case when the specifications are only over a portion of the frequency range.

- To determine the order of a FIR filter from phase (or equivalently from group delay) specifications. This problem has

Manuscript received March 18, 2005; revised May 13, 2006. The associate editor coordinating the review of this paper and approving it for publication was Dr. Behrouz Farhang-Boroujeny.

The authors are with the Department of Electrical and Electronic Engineering, Imperial College London, London SW7 2BT, U.K. (e-mail: a.constantinides@imperial.ac.uk; wen.li@imperial.ac.uk).

Color versions of one or more of the figures in this paper are available online at http://ieeexplore.ieee.org.

Digital Object Identifier 10.1109/TSP.2006.887565 not been addressed in the open literature. Two forms are examined, the first is concerned with the case when the specifications cover the entire frequency range $(0, \pi)$, while the second is involved with the partial case as defined under the item above.

- To determine the order of a FIR filter from magnitude and phase (or group delay) specifications. This case is a combination of the two above and is examined both for the complete and partial specifications.

The proposed approach is not only capable of dealing with specifications that go beyond the conventional formulas for the standard piece-wise constant magnitude FIR filter order estimation, but it also furnishes a nexus for order estimation in the new perspective from phase (or group delay) specifications. There are several formulas to aid the estimation of the digital filter order, which rely entirely on employing the gain specifications. These conventional methods make use of piece-wise filter specifications, i.e., the transition bandwidth as well as the passband and stopband ripple amplitudes [4], [5]. No algorithms have been described in the open literature which can estimate the FIR filter order from a general set of nonpiece-wise constant frequency response specifications, or, indeed, which enable the FIR filter order estimation from phase or group delay or some combinations of all independent specifications. In this paper, we propose an approach and develop viable algorithms for the digital filter order estimation problems above to be put into effect. The novel techniques open directions for further research and these directions are highlighted in the text as appropriate. The proposed approach in this paper is based on the use of root moments [6]-[10].

\section{ROOT MOMENTS}

A short description of root moments is given below for completeness. The general theory of homogeneous and symmetric functions of the roots of a polynomial is an area that received extensive attention in the past [6]-[8]. There are many homogeneous functions that can be formed in this context. Simplest amongst these are the first-order root moments, or simply the root moments of the polynomial, a concept directly related to the cepstrum in the signal processing domain. Other simple homogeneous functions of the roots can be formed such as the higherorder root moments and the Wronski moments [11] which apart from some creative, but limited use of second-order root moments in [12], have received little attention from a digital signal processing (DSP) perspective. In the present paper, we employ 
the first-order root moments. The standard first-order root moments have a classical formulation as follows. Given a set of coefficients $\left\{p_{i}\right\}$ of the following $n$th degree polynomial:

$$
f(x)=x^{n}+p_{1} x^{n-1}+p_{2} x^{n-2}+\cdots+p_{n}
$$

with roots $\left\{r_{i}\right\}, i=1, \ldots, n$, then the parameters $S_{m}$, below, are the first-order root moments of $f(x)$

$$
\begin{aligned}
S_{m} & =r_{1}^{m}+r_{2}^{m}+\cdots+r_{n}^{m}=\sum_{i=1}^{n} r_{i}^{m} \\
m & =0, \pm 1, \pm 2, \ldots
\end{aligned}
$$

Recently, the subject of root moments has attracted considerable attention from various perspectives, encompassing applications that include polynomial root finding procedures as in [13]-[17], FIR filter design [18], and FIR filter phase adjustment procedures as in [9], [11], [12], [19]. The wider potential of the root moment perspective on various aspects of digital filter design remains, in the main, an open and fertile field for further research. Precursor work that covers similar concepts includes the approaches based on cepstral techniques for digital filter design [20]-[22]. These papers describe in a very creative way, novel techniques concerning some aspects of digital filter design, but do not address the problem of order estimation, the subject of this paper.

\section{A. Root Moments and the Newton Identities}

There exists a simple relationship between the coefficients of the given polynomial and its root moments as follows [5], [6], [9]:

$$
\begin{aligned}
S_{m}+p_{1} S_{m-1}+p_{2} S_{m-2}+\cdots+m p_{m} & =0 \\
m & \leq n \\
S_{m}+p_{1} S_{m-1}+p_{2} S_{m-2}+\cdots+p_{n} S_{m-n} & =0 \\
m & >n
\end{aligned}
$$

with $S_{0}=n$.

These equations are known for $m \geq 1$ in Signal Processing in the context of cepstra [5], [10], [23], [24]. However, they are considerably older than that concept. They are also known in the mathematical theory of equations as the Newton identities, and essentially date back to the seventeenth century Scottish mathematician Gregory who first developed such relationships for polynomials of up to fifth degree [25]. The generalization of these relationships for any degree is due to the genius of Isaac Newton [6]. For our purposes, we note that given the set $\left\{p_{i}\right\}$ one can determine the set $\left\{S_{i}\right\}$, and conversely given $\left\{S_{i}\right\}$ one can determine $\left\{p_{i}\right\}$. Hence, the polynomial coefficients or the root moments can be used equivalently, as one set of parameters implies the other.

It is noteworthy to observe at this juncture the point aforementioned that the zero frequency cepstral component presents either some interpretational difficulties for nonpolynomial-type signals (e.g., signals evolving with time without a predetermined termination point), or it offers no additional information for the DSP problems in hand. Two cesptral forms have been used extensively in DSP. The real form which is based on the Fourier transform of the logarithm of the magnitude of the spectrum of a given signal. One difficulty associated with this form of the cepstrum, as is well known and pointed out typically for example in [26], is that it makes the transformation noninvertible. The second form of the cepstrum is the so-called complex, or differential form, which is based upon the Fourier transform of the differential of the logarithm of the complex spectrum (i.e., including both amplitude and phase components) as defined by Polydoros and Fam in [24]. Unfortunately, this approach will yield only the cepstrum of the minimum phase factor. In addition, it is standard practice in this context to set, by definition, the zero frequency value equal to zero [5], [21]. This assignment is unnecessary, and in the case of polynomials inappropriate, as their zero degree root moment (which essentially corresponds to the zero frequency value of the complex cepstrum) has a specific value equal to order of the polynomial under study. The use of the cepstral nexus for digital filter design with its conditioning, therefore, will inevitably be hindered by such definitional or traditional use of the cepstrum. However, the classical root moments provide an alternative formalism of precise, unambiguous, and invertible transformations of polynomials, and which is also amenable to further extensions within the broad context of higher-order symmetric functions, as witnessed by the second-order case in [12]. There are two useful results with respect to the use of root moments as follows. Let the root moments of polynomial $f_{1}(z)$ be $S_{m}^{f_{1}}$ and those of polynomial $f_{2}(z)$ be $S_{m}^{f_{2}}$. Then $S_{m}^{f}=S_{m}^{f_{1}}+S_{m}^{f_{2}}$ are the root moments of $f(z)=f_{1}(z) f_{2}(z)$, while $S_{m}^{g}=S_{m}^{f_{1}}-S_{m}^{f_{2}}$ are the root moments of $g(z)=f_{1}(z) / f_{2}(z)[12]$.

\section{B. Direct Computation of Root Moments: Cauchy Integral}

The Newton identities yield the root moments of the entire polynomial, thereby encompassing not only those roots that lie within the unit circle, but also those that are outside it. Thus, if there are any roots outside the unit circle then the root moments will grow exponentially. However, it is often the case that a specific factor of a given polynomial $f(z)$ is required, such as the minimum phase factor, and in this case its root moments can be determined through the use of complex variable theory. Thus, if we let a closed contour $\Gamma$ contain the roots of the required factor of the polynomial $f(z)$ then from the Cauchy residue theorem the root moments of this factor are given by [12]

$$
S_{m}^{\Gamma}=\frac{1}{2 \pi j} \oint_{\Gamma} \frac{f^{\prime}(z)}{f(z)} z^{m} d z
$$

where $m$ is a positive integer. When $\Gamma$ is the unit circle $|z|=$ 1 the above corresponds to the definition of the complex, or differential cepstrum, defined in [24] in which, in addition, $m$ is taken as integer $m \geq 1$. In further work reported in [12] the index $m$ is taken to be $m \geq 0$ and is thereby interpreting correctly the value of $S_{0}$. For our purposes $f(z)$ represents a FIR transfer function $H(z)$, which for the moment is assumed to have no zeros on $\Gamma$ and $h_{0} \neq 0$, takes the form

$$
\begin{aligned}
H(z) & =h_{0}+h_{1} z^{-1}+h_{2} z^{-2}+\cdots+h_{n} z^{-n} \\
& =K \cdot\left[\prod_{i=1}^{n_{1}}\left(1-\alpha_{i} z^{-1}\right)\right] \cdot\left[\prod_{i=1}^{n_{2}}\left(1-\beta_{i} z^{-1}\right)\right] \\
& =K \cdot N_{1}(z) \cdot N_{2}(z)
\end{aligned}
$$


where $K$ is a constant. We remove the constraint of no zeros on $|z|=1$ in Section IV. The quantities $\left|\alpha_{i}\right|<1, \forall i$ are the zeros of the minimum phase part $N_{1}(z)$, and $\left|\beta_{i}\right|>1, \forall i$ are the zeros of the maximum phase part $N_{2}(z)$ of the filter such that

$$
\begin{aligned}
& N_{1}(z)=\prod_{i=1}^{n_{1}}\left(1-\alpha_{i} z^{-1}\right) \\
& N_{2}(z)=\prod_{i=1}^{n_{2}}\left(1-\beta_{i} z^{-1}\right) .
\end{aligned}
$$

Equation (6) becomes

$$
S_{m}^{\Gamma}=\frac{1}{2 \pi j} \oint_{\Gamma}\left[-\frac{n}{z}+\sum_{i=1}^{n_{1}} \frac{1}{\left(z-\alpha_{i}\right)}+\sum_{i=1}^{n_{2}} \frac{1}{\left(z-\beta_{i}\right)}\right] z^{m} d z
$$

where $n=n_{1}+n_{2}$. It is clear from the above that when $\Gamma$ is the unit circle then from (12) for $m=0$ we obtain the number of zeros inside the unit circle, as pointed out by [12]. For $m>1$ we do not obtain the complete root moments of $H(z)$, but only those of its minimum phase factor $N_{1}(z)$, since only this factor will contribute to the residues within $\Gamma$.

It is noted here that in general the above relationships are also pertinent to any, and possibly nonminimum phase, z-transform of a block signal. Hence careful use of the differential cepstrum should be exercised, in that the root moments so obtained may not representative of the entire signal in view of the fact that its maximum phase factor is not obtainable for the given definitions.

It is possible, however, to gain access to the maximum phase factor of $H(z)$ if we let $m<0$, as shown later. Indeed, if we set $m=-\mu>0$ of the three terms under the integral in (12) only the third term will contribute nonzero residues as a typical element in the summation will be

$$
\frac{1}{z^{\mu}\left(z-\beta_{i}\right)}=\frac{1}{\beta_{i}^{\mu}} \frac{1}{z-\beta_{i}}-\frac{1}{\beta_{i}} \frac{\sum_{p=0}^{\mu-1}\left(\frac{z}{\beta_{i}}\right)^{p}}{z^{\mu}} .
$$

The first component on the right-hand side has zero contributions to the residue while the second component contributes a value of $-2 \pi j\left(1 / \beta_{i}^{\mu}\right)$. Therefore, the complex integral will yield the negative of the inverse root moments $-S_{-m}^{N_{2}}$ of the maximum phase factor of $H(z)$. With integer $m \geq 0$ we have the consequence, amongst others, that when $\Gamma$ is the unit circle we can estimate the root moments related only to the minimum phase factor of a given polynomial. As an example on the use of (6), we can extract that factor which contains the roots located on the unit circle as in Section IV. Following this procedure, we can reduce any FIR filter transfer function to the form given by (7).

\section{ROOT MOMENTS AND FUNDAMENTAL RELATIONSHIPS}

We assume at this juncture that the transfer functions we are considering have had any zeros on $|z|=1$ removed in a preprocessing step. Several procedures for doing so are described in Section IV, but, as these are dependent on the ensuing theory, we defer their description until we have provided a proper elaboration of the theory. In these circumstances, and assuming that $h_{0} \neq 0$, we can normalize the expression in (7) by dividing by its value at $\theta=0$. In these circumstances, the normalization has the effect, amongst others, of removing a possible phase discontinuity at $\theta=0$ due to any real positive zeros located outside the unit circle. Effectively, we shall have zero phase at zero frequency. A set of relationships can be derived connecting root moments and the magnitude and phase of a given FIR transfer function and are given by [10], [11], [27]-[29]

$$
\begin{aligned}
\ln A(\theta) & =\ln \tilde{K}-\sum_{m=1}^{\infty} \frac{S_{m}^{N_{1}}+S_{-m}^{N_{2}}}{m} \cos m \theta \\
\phi(\theta) & =-n_{2} \theta+\sum_{m=1}^{\infty} \frac{S_{m}^{N_{1}}-S_{-m}^{N_{2}}}{m} \sin m \theta .
\end{aligned}
$$

These equations are referred to as the Fundamental Relationships [11], [30] The corresponding form for the group delay is obtained by differentiating the phase expression to yield

$$
\tau(\theta)=n_{2}-\sum_{m=1}^{\infty}\left(S_{m}^{N_{1}}-S_{-m}^{N_{2}}\right) \cos m \theta .
$$

The corresponding inverse relationships are clearly obtained, for the amplitude and phase, as

$$
\begin{aligned}
S_{m}^{N_{1}} & =-\frac{m}{2 \pi} \int_{-\pi}^{\pi}\left[\ln \frac{A(\theta)}{\hat{K}} \cos m \theta-\hat{\phi}(\theta) \sin m \theta\right] d \theta \\
S_{-m}^{N_{2}} & =-\frac{m}{2 \pi} \int_{-\pi}^{\pi}\left[\ln \frac{A(\theta)}{\hat{K}} \cos m \theta+\hat{\phi}(\theta) \sin m \theta\right] d \theta
\end{aligned}
$$

where

$$
\hat{\phi}(\theta)=\phi(\theta)+n_{2} \theta .
$$

These relationships form the basis of our approach. Indeed, it is immediately apparent from (15) that we can derive the following results.

\section{Zeros on the Unit CIRCLE}

\section{A. A Preamble on Factor Isolation: Root Lassoing and Corralling}

We assumed earlier that the FIR transfer functions under study have no zeros on the unit circle. However, in some circumstances this can be too constraining. Here, we employ a root isolation and factor extraction, which reduces the specifications to the required form [11]. We begin by noting that if the FIR transfer function were known, then we can invoke the Argument Principle in relation to the formulation of the root moments in (6) to determine the number of zeros on the unit circle for $m=0$. For any $m>0$ we would obtain the higher degree root moments of the zeros located within $|z|=1$. Equation (6) can be used to isolate a factor that corresponds to roots which are located in a specific area, and thereby, without resorting to root finding procedures, we can extract this factor [19]. Specifically we can obtain that factor of $f(z)$ which has its zeros on the circumference of the unit circle. If the integration is carried out around the closed contour $\Gamma$, the root moments $S_{m}^{\Gamma}$ that 
TABLE I

PROCEDURE FOR ORDER ESTIMATION FROM ROOT MOMENTS

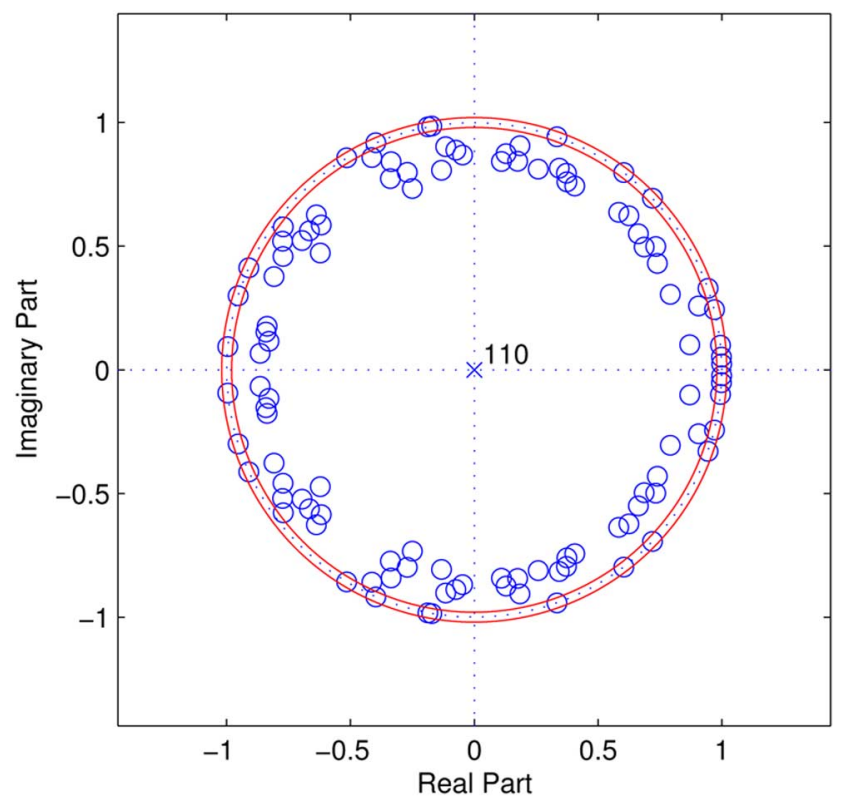

Fig. 1. Integration on the amplitude response alone, around the contour shown, to include roots that lie on the unit circle.

correspond to the roots inside the unit circle, are then obtained. From these root moments the factor of $f(z)$ having these zeros is obtainable by employing the Newton identities. This procedure makes it possible to obtain for a given polynomial $f(z)$ that specific factor that has its zeros in any desirable closed region on the z-plane, and this can be done without root finding techniques. This simple observation has been employed using the above formulation in [10] and [27], and further elaborated in [19], to produce minimum phase FIR filters. The contour in the general case need not be centered around the origin and need not be circular. This process of factor isolation, by an implicit root sieving or collection, is likened to a "rounding-up" process of freely roaming animals on the prairie, and can be described characteristically as lassoing and corralling the roots. It is an area for further study in relation to DSP applications.

For the purposes of the present publication the above procedure, suitably modified to circumvent the need to have available the FIR transfer function as assumed above, is used as a preprocessing step to isolate the zeros that are located on the unit circle. The results of Section IV-B show how such a zero isolation procedure can be achieved.

Anticipating these results we show in Fig. 1 a randomly generated real polynomial of order 110 with 30 zeros located in each of the regions inside $(0.80<$ radius $<0.97)$, and outside the unit circle $(1.10<$ radius $<1.20)$, and 50 zeros located on the unit circle (radius $=1$ ). The lassoing and corralling operations are carried out on the magnitude response of this polynomial alone. As expected the zeros on and inside the unit circle remain unchanged while those outside are reflected within the circle. We assume, henceforth, that such preprocessing operation has been carried out $a b$ initio. Subsequent to this preprocessing the FIR filters transfer function can be formulated as in (7).

\begin{tabular}{|l|l|}
\hline Step 1. & $\begin{array}{l}\text { Compute from appropriate equation the } \\
\text { desired root moments }\left\{S_{m}\right\} \text {. Set } n=1 ;\end{array}$ \\
\hline Step 2. & Evaluate $\widetilde{S}_{n+1}$ using Eqn(29) \\
\hline Step 3. & $\begin{array}{l}\text { Compare } \widetilde{S}_{n+1} \text { and } S_{n+1} \cdot \\
\text { If difference is zero or less than a threshold stop. } \\
\text { Else set } n=n+1 \text { and go to step 2. }\end{array}$ \\
\hline
\end{tabular}

In the above, however, the assumed availability of the FIR transfer function is not only premature but also infeasible. The problem specifications require that only the magnitude and possibly the phase (group delay) measurements should be employed. These are considered in Section IV-B.

\section{B. Estimating the Number of Zeros on $|z|=1$ From the Magnitude Response}

It is observed that if, in addition to any zeros inside and outside the unit circle, a polynomial $f(z)$ has zeros on the unit circle, then its minimum phase version has on the unit circle the same number of zeros in the same locations. Therefore, if we use the magnitude fundamental relationship, as in (14), to obtain the root moments these would correspond to a minimum phase polynomial in addition to whatever roots there may be on $|z|=1$. Thus an integration on a contour comprising two concentric circles centered at the origin with radii one slightly more than, and the other slightly less than unity, will furnish the root moments of the factor having its zeros on $|z|=1$. For a general case polynomial, the question of how close to unity the two radii must be, is a question that needs to be explored further. However, for equiripple transfer functions for piece-wise constant filter requirements, there are, in some cases, already sufficient indicators to make a reasonable selection [31].

Lassoing the roots as indicated above would produce the root moments which would be corralled by the Newton identities into a polynomial. Thus the nature of the resultant transfer function will be steered ultimately to the right form as required in (7). The required polynomial order would need to be determined from the techniques given in Section VI and specifically the procedure in Table I.

In the above, the logarithmic operation required in (14) will produce singularities in the case when the operations are realized using DFT and when a zero is located at a DFT position. It is possible to avoid such singularities through a series of alternative techniques and one of these is given later.

Let us add and subtract a small positive constant to the given gain measurements to produce a shifted version of the gain. The objective is then to use the root moments of this shifted version of the gain (which is strictly greater than zero, and hence its logarithm is finite) to estimate the root moments of the unshifted gain. We proceed as follows.

We write $A(\theta)=A(\theta)+\epsilon-\epsilon$ and, hence

$$
A(\theta)=A_{1}(\theta)\left(1-\frac{\epsilon}{A_{1}(\theta)}\right)
$$


where $A_{1}(\theta)=A(\theta)+\epsilon$, the shifted version of $A(\theta)$. Thus, we can write after expanding the appropriate logarithms and taking the first-order terms

$$
S_{m}^{A} \cong S_{m}^{A_{1}}+S_{m}^{B}
$$

where $S_{m}^{B}$ are the root moments of a transfer function $B(\theta)$ having a gain response as given

$$
B(\theta)=e^{-\epsilon / A_{1}(\theta)} .
$$

This gain is notional and is to be used for the purpose of refining the root moment estimates of the given magnitude response. The approximation can be further refined, if needed, by taking more terms in the logarithmic expansion related to (20). Thus, with second-order terms included we have

$$
B(\theta)=e^{-\epsilon / A_{1}(\theta)-\epsilon^{2} / 2 A_{1}^{2}(\theta)} .
$$

It is noted that for small values of $\epsilon$ it may be sufficient to ignore the adjustment related to the second term.

The results of Section V furnish another perspective. Indeed, this specific section indicates how the number of zeros that lie outside the unit circle may be estimated from the overall unwrapped phase response of a polynomial. Thus in any of the above methods we can shift the roots of an estimated polynomial, of whatever degree, by a small amount so that the roots that lie about the circumference of the unit circle are shifted to the region outside the circle. The amount of shift is dictated by the annulus of acceptance, defined as an annular region enclosing the unit circle and a user-definable width. The shifting of roots is carried out by replacing $z^{-1}$ by $(\rho z)^{-1}$ which is equivalent to adjusting the polynomial coefficients. Thus if the estimated polynomial is

$$
f(z)=f_{0}+f_{1} z^{-1}+f_{2} z^{-2}+\cdots+f_{n} z^{-n}
$$

then a polynomial having its roots shifted by $\rho$, and scaled for computational reasons, is

$$
\widetilde{f}(z)=\rho^{n} f_{0}+\rho^{n-1} f_{1} z^{-1}+\rho^{n-2} f_{2} z^{-2}+\cdots+f_{n} z^{-n} .
$$

The phase response of $\tilde{f}(z)$ can be used as indicated below in Section V to estimate the number of zeros that lie within the annulus of acceptance.

\section{Zeros Outside the Unit Circle}

On setting $\theta=\pi$ in (15) we obtain directly

$$
n_{2}=-\frac{\phi(\pi)}{\pi} \text {. }
$$

Thus, given the unwrapped phase response of a real transfer function the number of zeros that lie outside the unit circle is given from the total phase at the Nyquist frequency. This result can also be obtained from the Argument Principle [32].

Moreover, it is seen from (16) that by integrating over the interval $(-\pi, \pi)$, or indeed over one half of the interval $(0, \pi)$, the average group delay is also equal to the number of zeros located outside the unit circle. In a way, this observation is a restatement of the previous result in (24), but in practical applications it may be a useful indicator.

The computation involved in (24) requires the unwrapped phase response only at $\theta=\pi$. It is possible to use standard phase unwrapping algorithms but these will produce values at all frequencies. A simple approach is as follows. Let the wrapped phase response be $\widehat{\phi}(\theta)$ which exhibits discontinuities of $\pi$ radians. Then the variable $u(\theta)=\exp (j \widehat{\phi}(\theta))$ is continuous without these discontinuities. Moreover, $u(\theta)=\exp (j \phi(\theta))$. Thus, we can write

$$
\frac{d \phi(\theta)}{d(\theta)}=-j \bar{u}(\theta) \frac{d u(\theta)}{d(\theta)} .
$$

The variable $\bar{u}(\theta)$ is the complex conjugate of $u(\theta)$. Equation (25) can then be integrated as the phase unwrapping objective of the current type. This would require the estimation of the gradient $d u(\theta) / d(\theta)$. In a discrete realization we can estimate the gradient as $\left[u\left(\theta_{k}\right)-u\left(\theta_{k-1}\right)\right] /\left[\theta_{k}-\theta_{k-1}\right]$, the accuracy of which is controllable. In a DFT realization of the root moments the angular (frequency) difference is constant and equal to $2 \pi / N$ where $N$ is the DFT length. Then the unwrapped phase is estimated as the accumulation of

$$
\phi\left(\theta_{k}\right)=\phi\left(\theta_{k-1}\right)-j \bar{u}\left(\theta_{k}\right)\left[u\left(\theta_{k}\right)-u\left(\theta_{k-1}\right)\right]
$$

obtained from (25). In the problems under consideration, the phase response at zero frequency is equal to zero. Thus, summing the phase difference samples $\phi\left(\theta_{k}\right)-\phi\left(\theta_{k-1}\right)$ above, for $k=0, \ldots, N / 2$, is sufficient to estimate $\phi(\pi)$. The result is real for real systems. Thus, in practice one need only take the real part of the accumulation. The imaginary part is unlikely to be identically zero, and its value can serve as an indicator on the accuracy of the approximation employed.

\section{Zeros Inside the UnIT CIRCLE}

The estimation of the number of zeros inside the unit circle is not as straightforward. We can place some bounds on the value by considering the phase response of a companion all-pass filter for which we have already some results concerning the behavior of its phase response [33].

Let us consider a minimum phase polynomial $f(z)$ of order $n_{\min }$ and phase response $\phi_{\min }(\theta)$. Then

$$
A(z)=\frac{z^{-n_{\min }} f\left(z^{-1}\right)}{f(z)}
$$

is a stable all-pass function, the phase response of which is given by

$$
\phi_{A}(\theta)=-n_{\min } \theta-2 \phi_{\min }(\theta) .
$$

Consequently, $\phi_{A}(\theta)$ must satisfy the constraints for an allpass filter phase response, namely that it is a monotonically decreasing function of frequency. This result is based on the separation property of reactance functions as given in [33]. Hence, we obtain the result that a lower bound on the number of zeros inside the unit circle is given by

$$
n_{\min }>-2 \frac{d \phi_{\min }(\theta)}{d \theta} .
$$


The bound may not be very tight as it depends on the variation of the minimum phase function $\phi_{\min }(\theta)$. However, it does provide a guide on a possible choice, in the absence of any further information.

\section{A. Principles of Algebraic Order Estimation}

The estimation of the order is based upon the use of the root moments of a FIR digital filter transfer function. The significant point to observe is that the root moments can be derived from any one of the system function specifications such as the magnitude response, the phase response or, indeed, from the group delay or differential gain specifications. Moreover, an independent combination of these can yield the root moments of the minimum phase and maximum phase factors by means of the fundamental relationships [10], [27], [28]. Thus instead of estimating the order from the magnitude or phase specifications an equivalent problem can be posed in terms of root moments. The a priori desirable specifications relating to the transfer function $H(z)$ can lead to the following:

1) a minimum phase filter;

2) a maximum phase filter, or

3) a mixed phase filter.

The minimum phase case is the pivotal form of the problem in that the other two cases will be reduced to this form for their solution.

1) The Minimum Phase Case: The problem is as follows: Given a sequence of consecutive root moments $S_{m}^{f}$ of a minimum phase polynomial $f(z)$ in $z^{-1}$ to estimate the order of the polynomial. It is assumed that the length of the root moment sequence is greater that the order of the polynomial. In practice, this means that we estimate as many root moments $S_{m}^{f}$ from the specifications as we can. An algorithms is presented below. It relies on the use of the Newton identities and on the following observation. If the order of the FIR transfer function is $n$ then the Newton identities in (4) can be used to estimate the value of the root moment $S_{n+1}$. This root moment, obtainable from (5), combines all the root moments $\left[S_{1}, S_{2}, S_{3}, \ldots, S_{n}\right]$ and the estimated polynomial coefficients up to that point $\left[1, p_{1}, p_{2}, p_{3}, \ldots, p_{n}\right]$ as

$$
S_{n+1}=-\left[p_{1} S_{n}+p_{2} S_{n-1}+\cdots+p_{n} S_{1}\right] .
$$

The polynomial coefficients up to index $n$ are evaluated iteratively from the Newton identities in (4). Thus a complete enumeration procedure can be set up starting with $n=1$ and at each stage checking the evaluated next root moment against the corresponding value obtained from the specifications. If there is correspondence between these two values then the order has been determined.

In practice, it will be found that we need to test the correspondence for more than one value between the estimated and evaluated root moments. The procedure is described in Table I for ease of implementation.

In Table I, some further refinements can be implemented, as aforementioned, particularly in relation to the comparison procedure in Step 3. There are many ways of setting up stopping criteria, and it is an area for further study.

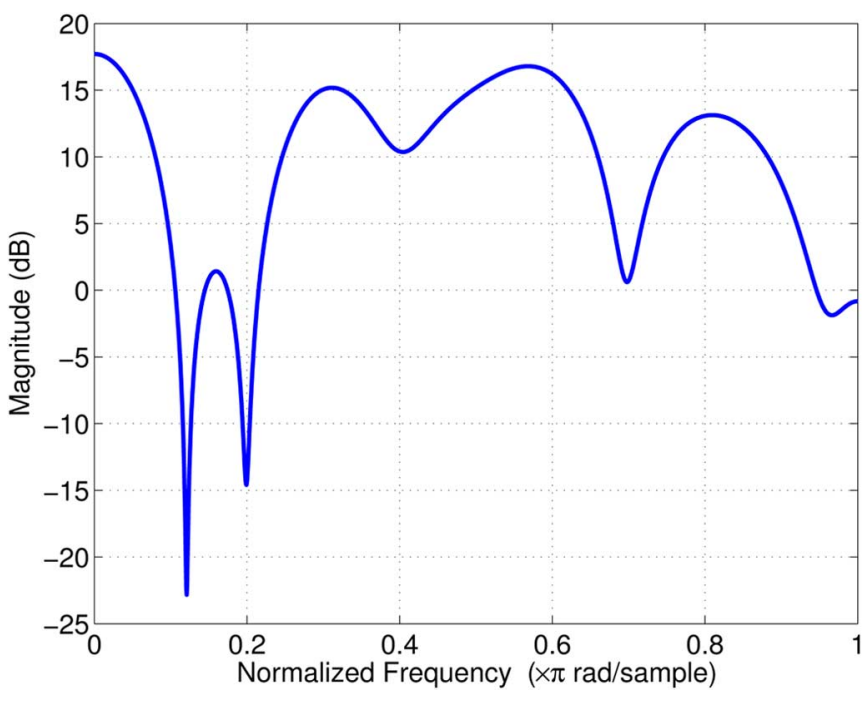

Fig. 2. Order estimation from complete magnitude response. The estimated order 15 is equal to the given filter order.

\section{B. Order From Magnitude Specifications Alone}

We are now in a position to pull together the strands for the solution of the digital filter order estimation problem as follows.

1) Magnitude and First-Order Root Moments: In view of the above, what is needed is the estimation of the root moments from the magnitude specifications. This can be done directly from (14), for example by taking the inverse Fourier transform. The operation can be implemented via a FFT routine. It should be noted that since no phase information is available in this present case, the computed root moments will be those of a minimum phase filter. With the root moments available we can use the procedure of Table I. This procedure is applied to a system for which the complete amplitude response is given as shown in Fig. 2. The estimated order is equal to 15 which is precisely the value of the original FIR system from which the response has been obtained. However, a more realistic situation is presented below. This, in fact, is the case that originally stimulated the recent study. The case in question corresponds to the problem of having measurements of gain over a partial range of frequency and requiring to produce a gain equalizer over this range.

2) Partial Magnitude and First-Order Root Moments: In the case when the magnitude is partially specified over a set of frequency values that do not cover the entire frequency range, the approach is similar to the complete case. It is possible to estimate the root moments from (14) for $m$ larger than the probable order of the transfer function, by treating the equation as a set of linear functional relationship for which the unknowns are $S_{m}$. Then the above procedure can be put into effect. However, it may be the case that too close a set of frequency values will ill-condition the linear problem, and hence the estimation may not be robust. This is likely to be the case when the given frequency range is narrow, the order is high, and the magnitude response features to be represented are too involved. The results of this procedure are given in Fig. 3. 


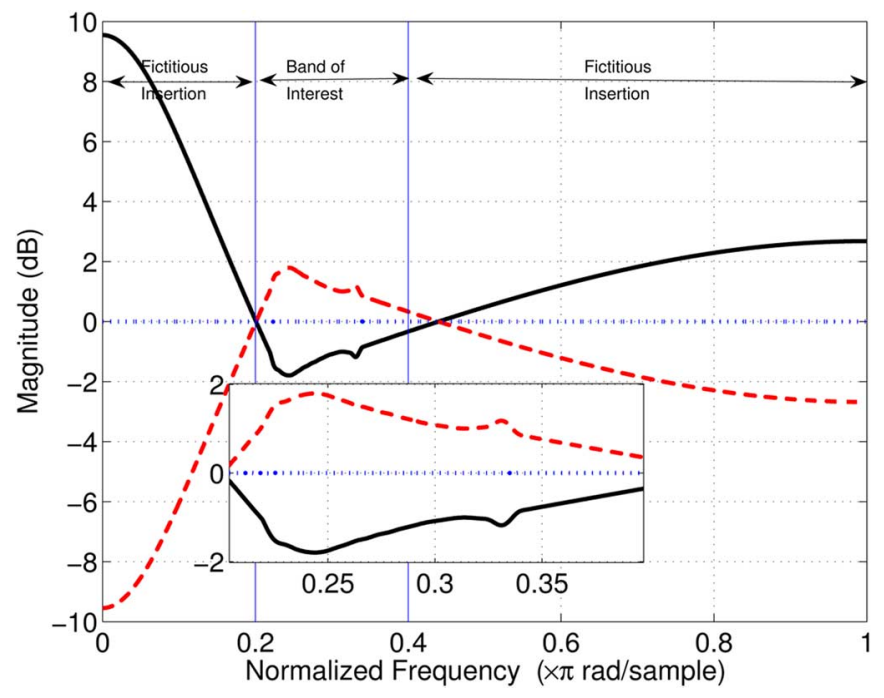

Fig. 3. Order estimation from partial magnitude measurements. Solid: Magnitude to be equalized with inserted fictitious response outside the required band. Dashdot: Equalizer's magnitude. The estimated order is 249. Dotted: Equalized overall magnitude response.

\section{Are the Magnitude Measurements From a FIR or IIR Filter?}

Let us assume that certain noiseless measurements have been carried out on an unknown FIR or IIR system, and that we have its magnitude response $A(\theta)$. In line with the results of Section VI, we can assume that zeros on the unit circle have been lassoed and corralled to give the factor containing these as a preprocessing step, and, hence, we can take $A(\theta)$ to be nonzero on $|z|=1$. A theoretical question can be posed as follows: Is it possible from these measurements alone to determine whether the unknown system is FIR or IIR?

To answer this question we observe that an equivalent question may be posed in terms of the root moments of the measured magnitude response. The problem in this form is tractable in that we can proceed to determine the order of a polynomial from its root moments. If the order found is finite, that is to say if the process terminates at a finite point, less than the order of the size of the FFT used in the computations, then the given specifications pertain to a FIR system. Otherwise the system is IIR. In practice, the measurements are inaccurate or contain noise, and hence a decision has to be made as to how close the root moment correspondence has to be before the process is considered to have terminated. Clearly this is a matter of setting up thresholds and related decision stopping criteria, and is a subject for further study.

\section{Order From Phase Specifications Alone}

1) Phase and First-Order Root Moments: When the given specifications are only with respect to the phase response then we have a direct benefit and some additional problems. Initially we obtain the benefit in that we can determine the number of zeros outside the unit circle required by the phase specifications as indicated by (24). Subsequently, the linear term corre-

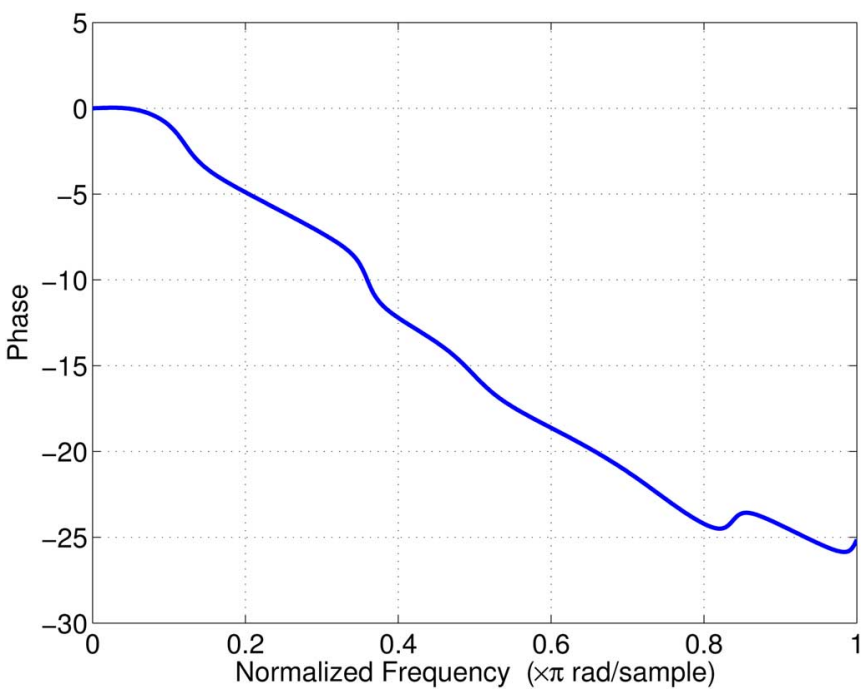

Fig. 4. Order estimation from complete phase response. The estimated order 15 is equal to the given filter order.

sponding to these in (15) can be removed to produce a modified phase response

$$
\widetilde{\phi}(\theta)=\sum_{m=1}^{\infty} \frac{S_{m}^{N_{1}}-S_{-m}^{N_{2}}}{m} \sin m \theta .
$$

In view of the difference operation in the root moments under the summation sign above, the expression may be construed as the phase response of a ratio of two polynomials

$$
G(z)=\frac{N_{1}(z)}{z^{-n_{2}} N_{2}(z)} .
$$

The numerator and denominator of the above are both minimum phase, the denominator being the reversed version of the maximum phase factor of the original polynomial transfer function. Thus, the problem becomes essentially the determination of a minimum phase IIR system from its phase response even though the initial specifications were for a FIR system.

We proceed as follows. The root moments of $\widetilde{\phi}(\theta)$ can be determined by using the phase form of the fundamental relationships. By taking the negative of these moments and using the Newton identities a polynomial transfer function can be derived. This transfer function $\widetilde{G}(z)$ would correspond to the product of $z^{-n_{2}} N_{2}(z)$ with the Taylor series expansion of $N_{1}(z)$. Now, let us write $\widetilde{G}(z)$ as a long polynomial

$$
\widetilde{G}(z)=g_{0}+g_{1} z^{-1} g_{2} z^{-2}+\cdots g_{N} z^{-N}=\frac{D(z)}{N_{1}(z)}
$$

where $D(z)=z^{-n_{2}} N_{2}(z)$.

We know that $D(z)$ is of order $n_{2}$ and, hence, by cross multiplying and equating powers on either side of (32) we can determine $N_{1}(z)$ as a long polynomial in $z^{-1}$. Its root moments can now be determined from the Newton identities and, hence, the test outlined in Table I can be applied to estimate the order $n_{1}$. Fig. 4 shows a nonminimum phase response from which the order of the FIR filter realizing this response is to be determined. 


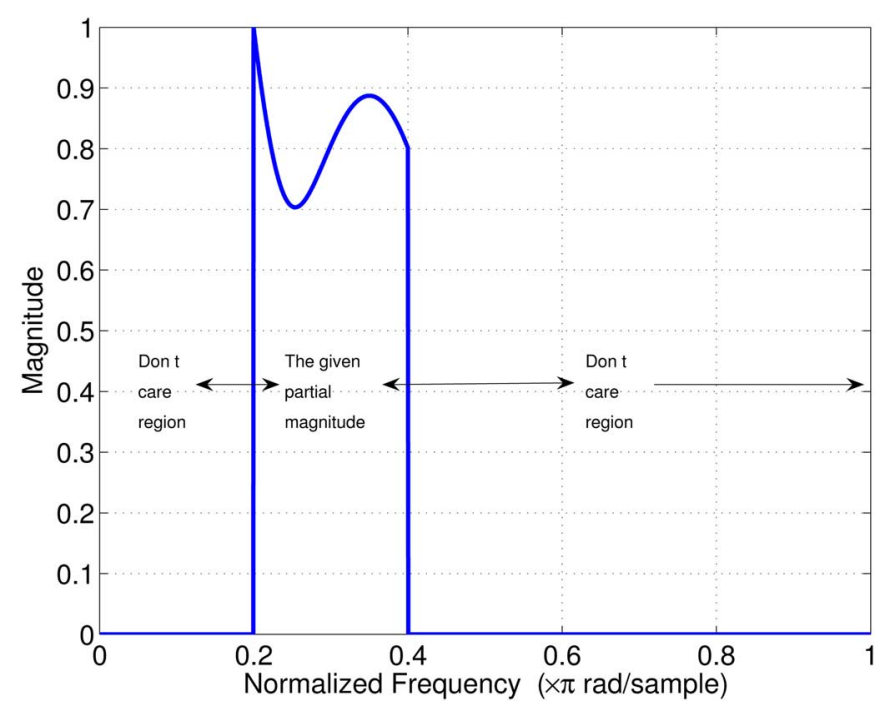

Fig. 5. Partial magnitude response from $0.2 \pi$ and $0.4 \pi$.

Using the results of this paragraph it found that there are 8 zeros outside the unit circle and the total order of the system is estimated to be 15 .

\section{E. Order From Joint Magnitude and Phase Specifications}

When the phase and magnitude specifications are jointly available then we have substantial information to help the process. However, the temptation to resort at this juncture to a naive use of the discrete Fourier transform (DFT) of the specifications should be resisted. The DFT length to be used in any nontrivial case will need to be long enough to accommodate the finer features in the specifications (i.e., undulations in the responses). Thus, the uncritical use of the DFT will produce a FIR filter length equal to the size of the DFT, which is quite inappropriate. More importantly, however, the specifications for gain and for phase may be partially defined, and may not be over the same range of frequencies, but over different, nonoverlapping and perhaps noncontiguous ranges. In such circumstances it is not even feasible to employ a DFT.

First-order root moments provide a framework for the estimation of the minimum phase and the maximum phase factors of the required transfer function as described here.

1) Joint Magnitude and Phase First-Order Root Moments: The fundamental relationships of (14) and (15) provide a direct estimation of the root moments $S_{m}^{N_{1}}$ of the minimum phase factor $N_{1}(z)$ and the inverse root moments $S_{-m}^{N_{1}}$ of the maximum phase factor $N_{2}(z)$ of the required FIR transfer function. This is clearly evident from the fact that while in (14) these moments appear as a sum in (15) appear as a difference, and, hence, they can be separately determined. In addition, the phase requirement furnishes the order $n_{2}$ of $N_{2}(z)$ in accordance with (24).

The final part in the estimation concerns the order of the minimum phase component. It is evident from the above that from the root moments $S_{m}^{N_{1}}$ we can estimate the order of the minimum phase factor $N_{1}(z)$ as outlined in Table I, thereby completing the task of estimating the total filter order.

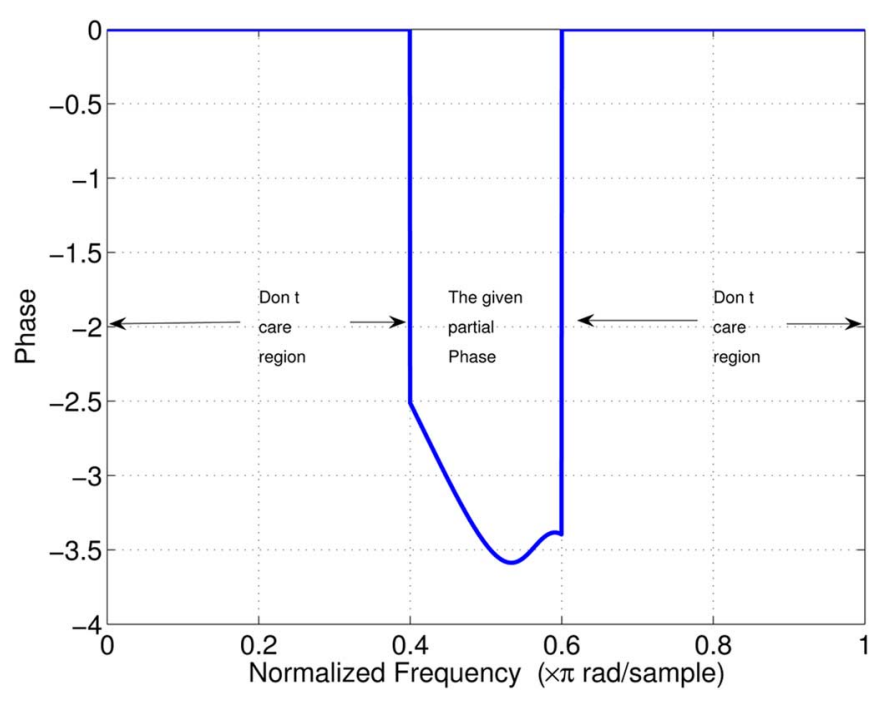

Fig. 6. Partial phase response from $0.4 \pi$ and $0.6 \pi$.

Figs. 5 and 6 show the partial magnitude and phase responses of system to be used in the order estimation procedure. The estimated FIR order is found to be 4 . Two zeros are found to be located inside the unit circle and two outside the unit circle.

\section{F. Order Estimation From Partial Specifications}

Partial specifications very often arise from practical measurements in which the gain and possibly phase or group delay are obtainable or are required for only a specific and limited ranges of frequencies. The ranges may be coincident, overlapping, contiguous or unrelated. In all of these cases, the fundamental relationships of (14) and (15) may be seen as interpolating functions for which we have a specific set of values (the given specifications) and from which we need to determine the unknown root moments. Thus, a set of linear equations may be formed. In these the quantities, $n_{1}$ and $n_{2}$ may be treated as part of the unknown parameter set, in which case they are required to be integer, or the can be left out of the set and hence the root moments are obtained. At this stage, we can follow the above procedures as appropriate. There is one further point, however, that must be observed. The regions over which the requirements are unspecified, may be treated as "don't care regions" in which fictitious responses may be inserted. The insertions will need to be done in a way consistent with the magnitude and phase requirements at the fixed points as explained in [32]. The insertions facilitate the estimation process and render the problem better conditioned.

\section{EFFECTS OF NOISE}

It is assumed implicitly so far that the measurements on either gain or phase are made in a noiseless environment and in the absence of measurement uncertainty. However, this is an idealized condition since in practice the measurements will be corrupted by both environment noise and measurement uncertainty. In both cases, we assume that there are no systematic errors and that the deviations from the noiseless case are zero mean with symmetric probability density functions. 


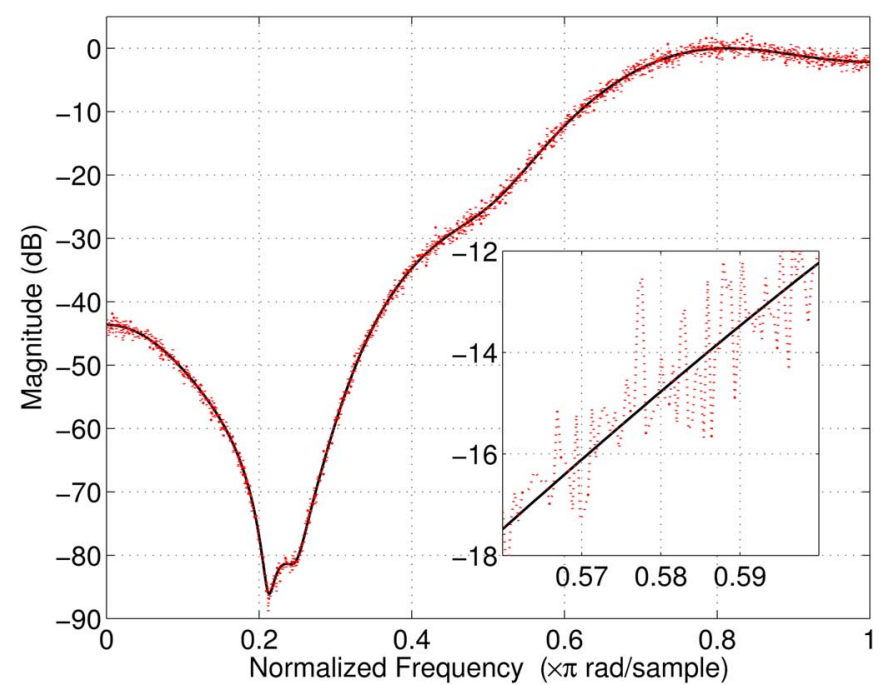

Fig. 7. Order estimation from noisy magnitude response. Solid: Noiseless magnitude response. Dotted: Noisy magnitude response with SNR $20 \mathrm{~dB}$.

\section{A. Noise in Gain}

Let the gain measurements be carried out in the logarithmic domain with noise component $\epsilon_{A}(\theta)$ which is zero mean with symmetric probability density function. The measured logarithmic gain is then assumed to be given by

$$
\log (\widetilde{A}(\theta))=\log (A(\theta))+\epsilon_{A}(\theta) .
$$

Therefore, the estimated root moments $S_{m}$ will be

$$
\widetilde{S}_{m}=S_{m}+\frac{m}{2 \pi} \int_{-\pi}^{\pi}\left[\epsilon_{A}(\theta) \cos m \theta\right] d \theta
$$

It is expected that the averaging effect in the second term related to noise is likely to have a reduced influence. However, in order that a more precise estimation of the overall influence is currently under study. The approach is to employ the Chebyshev inequality to estimate the probability that the error is bounded in a desired way. For the purposes of the current paper, we rely on the formulation. In Fig. 7, we show the gain with noise. The measured data-to-noise is taken to be $20 \mathrm{~dB}$ (with the mean data value removed). The estimated order in the noiseless case is found to be 20 . The estimated order with noise is determined as 20 .

\section{B. Noise in Phase}

In a similar way, the additive noise and uncertainty components in the phase will be expressed as additive disturbances to the overall root moment estimates as with the case of gain. Thus, the expected estimates are not substantially disturbed. However, the overall area is currently under extensive study as mentioned earlier. Fig. 8 shows a typical noise contaminated phase response. The estimation of order for this case having noise at 18 $\mathrm{dB}$ gives the figure of 8 as opposed to 20 for the noiseless case.

In a parallel study the use of the Poisson relationships [5] are employed as estimators of gain and phase. These are used as a preamble to applying the order estimation. The estimated order for the same phase example as above is found to be 20 .

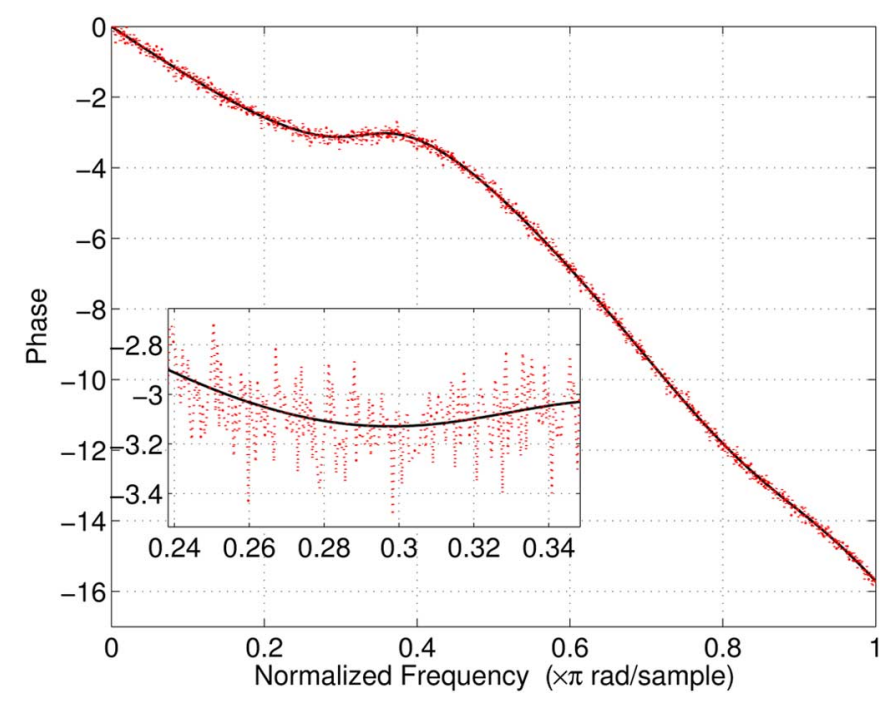

Fig. 8. Order estimation from noisy phase response. Solid: Noiseless phase response. Dotted: Noisy phase response with SNR $18 \mathrm{~dB}$.

\section{CONCLUSION}

In this paper, we set out the principles of estimating algebraically the order of a FIR digital filter from its, possibly partially defined, magnitude and gain measurements or specifications. The approach taken relies on the use of root moments. The overall framework is essentially algebraic in nature. An additional aim of the paper is to indicate further areas of research and these are highlighted in the text. The developed procedures offer opportunities for enhancing the methods available in this area and provide solutions to hitherto unexamined areas such as the FIR transfer function order estimation from phase specifications. Several examples are given to illustrate the principles. The results presented in this paper are currently extended to cover additional ideas related to the new concept of fractional root moments [11]. An interesting, and as yet unexplored, area is the extension of these concepts to multi-input multi-output systems. The algorithms described in the paper are available in MATLAB and can be downloaded from http://www.commsp.ee.ic.ac.uk/ people/agc.html.

\section{REFERENCES}

[1] J. F. Kaiser, "Nonrecursive digital filter design using the io-sinh window function," in Proc. 1974 IEEE Int. Symp. Circuits Syst., San Francisco, CA, Apr. 1974, pp. 20-23.

[2] O. Herrmann, L. R. Rabiner, and D. S. K. Chan, "Practical design rules for optimum finite impulse response lowpass digital filters," Bell Syst. Tech. J., vol. 52, pp. 769-799, 1973.

[3] L. R. Rabiner and R. W. Schafer, Digital Processing of Speech Signals, ser. Prentice-Hall Signal Processing. Englewood Cliffs, NJ: PrenticeHall, 1978.

[4] S. K. Mitra, Digital Signal Processing: A Computer-Based Approach. New York: McGraw-Hill, 1998.

[5] A. V. Oppenheim and R. W. Schafer, Discrete-Time Signal Processing, second ed. Upper Saddle River, NJ: Prentice-Hall, 1999.

[6] I. Todhunter, Theory of Equations. London, U.K.: Macmillan, 1882.

[7] E. Artin, Galois Theory, 2nd ed. South Bend, IN: Univ. Notre Dame Press, 1966.

[8] D. S. Dummit, Abstract Algebra, 2nd ed. Upper Saddle River, N.J: Prentice-Hall, 1999.

[9] T. Stathaki, "Cumulant-based and algebraic techniques for signal modelling,” Ph.D. dissertation, Imperial College, London, U.K., 1994. 
[10] T. Stathaki and A. G. Constantinides, "Root moments: An alternative interpretation of cepstra for signal feature extraction and modelling," in 1995 Conf. Rec. 29th Asilomar Conf. Signals, Syst. Comput., Nov. 1995, vol. 2, pp. 1477-1481.

[11] W. M. Li, "Root moments and digital filter design," Ph.D. dissertation, Imperial College London, London, U.K., 2004.

[12] T. Stathaki, "Root moments: A digital signal-processing perspective," in Inst. Elect. Eng. Proc. Vision, Image and Signal Process., Aug. 1998, vol. 145 , pp. 293-302.

[13] L. Hoteit, "FFT-based fast polynomial rooting," Electron. Lett., vol. 35, pp. 965-966, Jun. 1999.

[14] - "FFT-based fast polynomial rooting," in Proc. IEEE Int. Conf. Acoust., Speech, Signal Process., 2000 (ICASSP), Jun. 2000, vol. 6, pp. 3315-3318.

[15] G. A. Sitton, C. S. Burrus, J. W. Fox, and S. Treitel, "Factoring veryhigh-degree polynomials," IEEE Signal Process. Mag., vol. 20, pp. 27-42, Nov. 2003.

[16] D. S. Huang and H. H. S. Ip, "Finding the maximum modulus roots of polynomials based on constrained neural networks," in IEEE Int. Conf. Acoust., Speech, Signal Process., Apr. 2003, vol. 2, pp. II-797.

[17] D. S. Huang, H. S. Ip, and H. Z. Chi, "A neural root finder of polynomials based on root moments," Neural Computat., vol. 16, pp. 1721-1762, 2004.

[18] N. Damera-Venkata, B. L. Evans, and S. R. McCaslin, "Design of optimal minimum-phase digital FIR filters using discrete Hilbert transforms," IEEE Trans. Signal Process., vol. 48, no. 5, pp. 1491-1495, May 2000.

[19] I. Fotinopoulos and T. Stathaki, "Equiripple minimum phase FIR filter design from linear phase systems using a novel technique for polynomial factorisation," in IEEE Global Telecommunications Conf. (IEEE GLOBECOM), 2000, vol. 3, pp. 1659-1663.

[20] S. Imai, "Digital filter design in the quefrency domain," in IEEE Trans. Acoust., Speech, Signal Process., Jun. 1978, vol. ASSP-26, pp. 226-235.

[21] G. A. Mian and A. P. Nainer, "A fast procedure to design equiriple minimum-phase FIR filtes," IEEE Trans. Circuits Syst., vol. CAS-29, no. 5, pp. 327-331, 1982.

[22] S.-C. Pei and S.-T. Lu, "Design of minimum-phase FIR digital filters by differential cepstrum," IEEE Trans. Circuits Syst., vol. CAS-33, pp. 570-576, May 1986.

[23] B. P. Bogert, M. J. R. Healy, and J. W. Tukey, M. Rosenblatt and Sons, Eds., "The quefrency alanysis of time series for echoes: Cepstrum, pseudo-autocovariance. Cross-cepstrum, and saphe cracking," in Proc. Symp. Time Series Analysis, New York, 1963, pp. 209-243.

[24] A. Polydoros and A. T. Fam, "The differential cepstrum definition and properties," in Proc. IEEE Int. Symp. Circuits Syst., Apr. 1981, pp. 77-80.

[25] E. T. Whittaker, The Calculus of Observations, 2nd ed. London, U.K.: Blackie and Sons, 1929.

[26] J. G. Proakis, C. M. Rader, F. Ling, and C. L. Nikias, Advanced Digital Signal Processing. New York: Macmillan, 1992.

[27] T. Stathaki, A. G. Constantinides, and G. Stathakis, "Minimum phase FIR filter design from linear phase systems using root moments," in Proc. 1998 IEEE Int. Conf. Acoust., Speech Signal Process. (ICASSP), May 12-15, 1998, vol. 3, pp. 1317-1320.

[28] T. Stathaki and I. Fotinopoulos, "Equiripple minimum phase FIR filter design from linear phase systems using root moments," IEEE Trans. Circuits Syst. II, Analog Digit. Signal Process., vol. 48, pp. 580-587, Jun. 2001

[29] A. G. Constantinides and W. M. Li, "Root moments and minimum phase FIR filter design," IEEE Trans. Signal Process., 2004, submitted for publication.
[30] A. G. Constantinides, "Digital signal processing and digital filters," in M.Sc. Course Lecture Notes. London, U.K.: Elect. Electron. Eng. Dept., Imperial College London, 2004.

[31] I. Fotinopoulos, T. Stathaki, and A. G. Constantinides, "A method for FIR filter design from joint amplitude and group delay characteristics," in Conf. Rec. 35th Asilomar Conf. Signals, Syst. Comput., 2001, vol. 1, pp. 621-625.

[32] A. G. Constantinides and W. M. Li, "A framework for the design of digital filters and equalizers from partial specifications based on root moments," IEEE Trans. Signal Process., 2004, submitted for publication.

[33] A. G. Constantinides, "Frequency functions-a class of real functions lowpass digital filters having equiripple passbands," in Proc. IEEE Int. Symp. Circuit Theory, 1970, pp. 134-135.

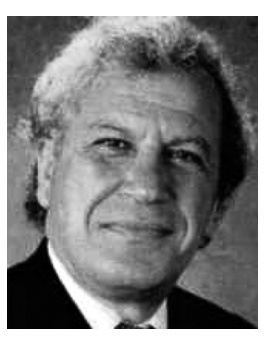

Anthony G. Constantinides (S'68-M74-SM'78F'98) is the Professor of Signal Processing at Imperial College London, U.K. He has been actively involved with research in various aspects of digital filter design, digital signal processing, and communications for more than 40 years. His recent work has been directed toward the demanding signal processing problems arising from the various areas of telecommunication. This work is supported by research grants and contracts from various government and industrial organizations. He has published several books and papers in learned journals in the area of digital signal processing and its applications.

Prof. Constantinides has served as the First President of the European Association for Signal Processing (EURASIP). He has been on, and is currently serving as, a member of many Technical Program Committees of the IEEE, the IEE, and other international conferences. He has organized the first-ever international series of meetings on Digital Signal Processing, in London initially in 1967, and in Florence. Italy, since 1972. In 1985, he was awarded by the French government the National Honour Chevalier, Palmes Academiques, and in 1996, received the Officier, Palmes Academiques. He received honorary doctorates, and holds Visiting Professorships, Distinguished Lectureships, Fellowships, and other honors internationally. He served as a Member of the Board of Governors of the IEEE Signal Processing Society, a member of several Technical Committees of the IEEE and the IEE, and is on the Editorial Board of the Proceedings of the Royal Society, and on several professional journals. He is a Fellow of the Royal Academy of Engineering, the IEE U.K., and an honorary member of Eta Kappa Nu.

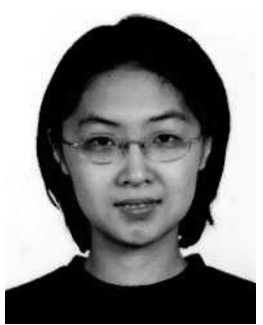

Wen Min Li was born in Harbin, China. She received the B.Sc. degree (first class honors) from the University of Central Lancashire, U.K., in 2000 and the M.Sc. degree in electrical and electronic engineering from Imperial College London, U.K., in 2001.

She is currently pursuing the Ph.D. degree at Imperial College London. Her research interests are in the areas of filter design and optical signal processing.

Ms. Li was awarded the Institution of Incorporated Engineers (IIE) Institution Award for outstanding performance in 2000 . 